\title{
Quantification of the effect of osteolytic metastases on bone strain within whole vertebrae using image registration
}

\author{
Hardisty M. R., Akens M. K., Hojjat S. P., \\ Yee A., and Whyne C. M.
}

Version Post-Print/Accepted Manuscript

Citation Hardisty MR, Akens MK, Hojjat SP, Yee A, Whyne CM. Quantification (published version) of the effect of osteolytic metastases on bone strain within whole vertebrae using image registration. J Orthop Res. 2012 Jul;30(7):1032-9. doi: 10.1002/jor.22045. PMID: 22213180

Publisher's Statement This is the peer reviewed version of the following article: Hardisty MR, Akens MK, Hojjat SP, Yee A, Whyne CM. Quantification of the effect of osteolytic metastases on bone strain within whole vertebrae using image registration. J Orthop Res. 2012 Jul;30(7):1032-9, which has been published in final form at https://dx.doi.org/10.1002/jor.22045. This article may be used for non-commercial purposes in accordance with Wiley Terms and Conditions for Self-Archiving.

How to cite TSpace items

Always cite the published version, so the author(s) will receive recognition through services that track citation counts, e.g. Scopus. If you need to cite the page number of the TSpace version (original manuscript or accepted manuscript) because you cannot access the published version, then cite the TSpace version in addition to the published version using the permanent URI (handle) found on the record page. 


\title{
Quantification of the Effect of Osteolytic Metastases on Bone Strain within Whole Vertebrae using Image Registration
}

\author{
${ }^{1}$ M.R. Hardisty MASc, ${ }^{2}$ M.K. Akens PhD, ${ }^{1}$ S.P. Hojjat MSc, ${ }^{2}$ A. Yee MSc MD FRCSC, ${ }^{1}$ C.M. \\ Whyne+ PhD \\ ${ }^{1}$ Orthopaedic Biomechanics Laboratory, \\ ${ }^{2}$ Department of Orthopaedic Surgery, \\ Sunnybrook Health Sciences Centre, \\ University of Toronto, \\ Toronto, $\mathrm{ON}$
}

\begin{abstract}
Orthopaedic Biomechanics Laboratory
Sunnybrook Health Sciences Centre

Postal Address:

Orthopaedic Biomechanics Laboratory

Sunnybrook Health Sciences Centre

2075 Bayview Ave. Room UB-19

Toronto, Ont.

M4N 3M5

Canada

Phone Number: 416-480-5056

Fax Number: 416-480-5856

+Email: Cari.whyne@sunnybrook.ca
\end{abstract}




\section{Abstract}

\section{Introduction}

The vertebral column is the most frequent site of metastatic involvement of the skeleton with up to $1 / 3$ of all cancer patients developing spinal metastases. Longer survival times for patients, particularly secondary to breast cancer, have increased the need for better understanding the impact of skeletal metastases on structural stability.

\section{Methods}

This study aims to apply image registration to calculate strain distributions in metastatically involved rodent vertebrae utilizing $\mu \mathrm{CT}$ imaging. Osteolytic vertebral lesions were developed in $5 \mathrm{rnu} / \mathrm{rnu}$ rats 2-3 weeks post intracardiac injection with MT-1 human breast cancer cells. An image registration algorithm was used to calculate and compare strain fields due to axial compressive loading in metastatically involved and control vertebrae.

\section{Results}

Tumour-bearing vertebrae had greatly increased compressive strains, double the magnitude of strain compared to control vertebrae $(p=0.01)$. Qualitatively strain concentrated within the growth plates in both tumour bearing and control vertebrae. Most interesting was the presence of strain concentrations at the dorsal wall in metastatically involved vertebrae, suggesting structural instability.

\section{Conclusions}

Strain distributions, quantified by image registration were consistent with known consequences of lytic involvement. Metastatically involved vertebrae had greater strain magnitude than control vertebrae. Strain concentrations at the dorsal wall in only the metastatic vertebrae, were consistent with higher incidence of burst fracture secondary to this pathology. Future use of image registration of whole vertebrae will allow focused examination of the efficacy of targeted and systemic treatments in reducing strains and the related risk of fracture in pathologic bones under simple and complex loading. 


\section{Introduction}

Sixty-five percent of patients with metastatic cancer have skeletal disease ${ }^{1}$, with up to $1 / 3$ of all cancer patients developing spinal metastases. ${ }^{2,3}$ Longer survival times for patients with metastatic bone disease have increased the risk of pathologic fracture. Skeletal metastases may be treated by systemic therapies (i.e. bisphosphonates), local minimally invasive approaches (i.e radiation therapy) or surgical stabilization. ${ }^{4-6}$ Development of new therapies that target skeletal metastases requires understanding of the biomechanical effects of the tumour within bone, as well as how effective new and existing therapies are at mitigating these consequences.

Much remains to be learned about how bones naturally experience load and how load distributions differ in the presence of osteolytic disease. Further elucidation of the mechanical environment of bony tissue affected by osteolytic disease could identify sites and mechanisms of fracture risk, detect possible sites of remodelling in a metastatic consequence, as well as provide information on the effectiveness of systemic or spatially focused treatments designed to have a mechanically stabilizing effect.

Non-invasive strength assessment of metastatically involved vertebrae has been performed using finite element analysis (FEA) and analytical calculations (Engineering Beam Theory) based on CT images. ${ }^{4-10}$ However, estimates of stress and apparent properties based on such modelling approaches are limited without apriori knowledge of material properties and when the underlying structures are not well understood. In contrast, image registration, can be used to calculate strain fields with no assumptions about structure or material behaviour. As such, image registration may be particularly useful for non-invasive assessment of skeletal pathology and new therapies, which may affect both structure and material properties of the bone and soft tissues.

Application of image registration to measure strain in whole bones is essential to properly characterize the physiologic and pathologic strain environments. Previous work has applied image registration to measure strain fields within bone and study its mechanics; however such methods have been quite limited in application and development in 3D. ${ }^{11-15}$ Previous 3D studies have focused on the mechanical workings of trabecular bone cores ${ }^{13-15}$ however results from experiments may suggest premature fracture and abnormally high non-physiological strain patterns as they remove bone from its natural mechanical support system. ${ }^{16,17}$ 3D image registration methods have previously been used to look at the effect that subtle changes in fusion cage designs had upon strain distributions within the adjacent vertebrae in an in vitro biomechanical study. ${ }^{18}$

In our work to date we have developed and validated an intensity matching image registration method to accurately measure and spatially resolve strain in $3 \mathrm{D}$ in whole bones using $\mu \mathrm{CT}$ imaging. ${ }^{19,20}$ We propose to utilize this algorithm to qualitatively and quantitatively assess changes in mechanical behaviour due to metastatic involvement of vertebrae. It is hypothesized that strain distributions measured by image registration will occur in a manner that is consistent with known fracture patterns in the metastatic spine. Specifically, this study aims to apply image registration to calculate and characterise the strain distributions in metastatically involved rodent vertebrae utilizing $\mu \mathrm{CT}$ imaging. 


\section{Materials and Methods}

\section{Metastatic Tumour Model}

A previously described model of osteolytic vertebral metastasis was used to examine the biomechanical effects of tumour involvement within the bony spine. ${ }^{21}$ The study was approved by the Sunnybrook Health Sciences Centre Animal Care Committee. MT-1 human breast cancer carcinoma cells $\left(2 \times 10^{6} / 200 \mu \mathrm{l}\right)$ transfected with the luciferase gene were injected intracardially under general anaesthesia ( $2 \%$ isofluorane in oxygen) into 5 immunocompromised nude $\mathrm{rnu} / \mathrm{rnu}$ female rats (5-6weeks of age) (Harlan, Indianapolis, USA). Injections were made into the left ventricle using a $1 \mathrm{ml}$ syringe with a $26 \mathrm{~g}$ needle. After injection the rats received analgesic (buprenorphine, $0.03-0.05 \mathrm{mg} / \mathrm{kg}$ ) and were put back into their cages with free access to food and water. The rats were monitored daily to ensure health and normal behaviour during the study.

Bioluminescent imaging was done to confirm the presence of and to localise the tumour within the bony spine, beginning fifteen days after injection. Rats received an intra-peritoneal injection of luciferin $(60 \mathrm{mg} / \mathrm{kg})$ dissolved in saline to facilitate bioluminescence scanning (Xenogen Inc, Alameda, USA) under general anaesthesia. Rats were sacrificed once the presence of a tumour was verified (2-3 weeks post injection). To identify the vertebral level with the greatest tumour involvement, the location of maximum bioluminescent signal was identified on faxitron images (Faxitron X-Ray Corporation, Wheeling, USA) by insertion of a needle guided by the bioluminescent imaging. Vertebrae with the highest level of metastatic involvement were excised within a three-level motion segment. For comparison purposes the tumour-bearing vertebral motion segments were matched with healthy vertebral motion segments from similarly aged control animals (i.e. a tumour-bearing L2 vertebra was matched with an L2 vertebra from a non-pathologic animal creating a balanced design, $n=5$ in each group).

\section{Loading and imaging of spines}

All 10 specimens were $\mu \mathrm{CT}$ scanned (GE Explore Locus, General Electric Company, Fairfield, USA) under an x-ray source at $90 \mu \mathrm{A}$ and $80 \mathrm{kV}$, with 907 views covering $360^{\circ}$ of rotation, in both unloaded and loaded configurations. The three-level motion segments were loaded in vitro in axial compression within a custom-built force-calibrated CT compatible loading device. (Figure 1) Axial compressive loads were applied to each metastatically involved spinal motion segment (pre-failure loading of 44-67N) and its matched control. Load magnitude was decided upon by estimating the stability of the tumour bearing spinal motion segment, relying upon our previous studies of loading rat motion segments. ${ }^{20}$ The load magnitude applied to the tumour bearing vertebrae was replicated in the spinal level matched healthy control vertebrae. Reconstruction of the volumes was done to $17.5 \mu \mathrm{m} / \mathrm{voxel}$; the exact image size reconstructed varied from scan to scan based upon the size and orientation of the region of interest. The unloaded configuration of the specimen was imaged while immersed in agar to prevent motion.

\section{Assessment of metastatic involvement}

Due to the infiltrative nature of the metastatic involvement of the MT-1 tumours within the vertebrae, the extent of lytic involvement (bone resorption) was assessed by quantifying differences in trabecular bone density. Average bone density within the vertebral bodies of the 
control and tumour-bearing vertebrae were compared based on automated volumetric $\mu \mathrm{CT}$ measurements. ${ }^{22,23}$ (Amira 3.1.1, Visage Imaging, San Diego, USA)

\section{Comparison of strains}

Image registration was used to measure strain in each vertebra under load. Strain was measured exclusively within the vertebral body. The vertebral body is the primary load bearing structure and contains the majority of the trabecular bone essential for proper image correlation. Strain was quantified within each specimen by a previously described and validated multiresolution image registration routine. ${ }^{19}$ Briefly, each whole loaded scan was registered to its associated unloaded scan. The unloaded scan was then divided into eight equally sized pieces that were individually registered to the loaded scan using the previous registration as an initial best guess. Each of the eight pieces were then further divided into 8 additional sub-pieces and reregistered in an ongoing pyramid scheme through four levels of refinement. Any outer region that did not contain bone was removed by manual segmentation of the $\mu \mathrm{CT}$ volume. Each individual registration used a regular step gradient descent optimisation of an affine mapping (T), permitting shearing, rotation, scaling and translation (12 DOF, Equation 1a), relying in the ITK 1.6.0 library. (NLM, Bethesda, MD, USA) Mutual Information was used as the similarity metric. The displacement of the center of each sub region that was registered was calculated from the affine transform found. The displacement field (A) was then used to calculate the strains (e) field. (Equations 1b, c) The size of the smallest individual sub-regions in which strain was resolved varied from $385 \times 542 \times 297.5 \mu \mathrm{m}$ to $402.5 \times 612.5 \times 490 \mu \mathrm{m}$, depending upon the exact size of the scan. The regions varied with the size of the vertebrae imaged (larger vertebrae had larger individual regions in which strain was resolved). The size of these regions was determined based upon our previous work to validate this method within the rnu/rnu rat model used in this investigation. ${ }^{19}$ The size of these individual regions was what determined the spatial accuracy of the strain field calculated below. For comparison, previous work by our group has found trabecular thickness to be on average $90 \mu \mathrm{m}$ and trabecular spacing to be on average $150 \mu \mathrm{m} .^{24}$

$$
\begin{gathered}
\mathrm{T}=\left[\begin{array}{ccc|c}
J_{11} & J_{12} & J_{13} \mid & X_{\text {Trans }} \\
J_{21} & J_{22} & J_{23} \mid & Y_{\text {Trans }} \\
\underline{J_{31}} & \underline{J_{32}} & \underline{J_{33}} & Z_{\text {Trans }} \\
\hline X_{\text {Centre }} & Y_{\text {Centre }} & Z_{\text {Centre }} & 1
\end{array}\right] \\
\vec{A}(x, y, z)=\tilde{T} P(x, y, z)-P(x, y, z) \\
e=\frac{1}{2}\left(\nabla \vec{A}^{T}+\nabla \vec{A}\right)
\end{gathered}
$$

$\mathrm{T}$ is the affine mapping describing the deformation between the loaded and unloaded sub regions within each scan, $\mathrm{J}_{\mathrm{ij}}$ are the elements of the affine matrix that contain all the information causing scaling, rotation and shearing. $\left(\mathrm{X}_{\text {Trans }}, \mathrm{Y}_{\text {Trans }}, \mathrm{Z}_{\text {Trans }}\right)$ represent the translation of the region and $\left(\mathrm{X}_{\text {Centre }}, \mathrm{Y}_{\text {Centre }}, \mathrm{Z}_{\text {Centre }}\right)$ is the original centre of the region about which the mapping is done. The Displacement (A) was calculated through the vector field, defined by finding the displacements between the centers of the subregions $(\mathrm{P})$ that are individually registered. The displacement fields were smoothed with a lanczos filter prior to calculating strain. (Amira 3.1.1) The strains (e) quantified by evaluating the gradient and calculating the infinitesimal strain tensor. 
The axial strain of the vertebrae was used as the primary outcome variable in this analysis as it will yield the largest strain values experienced by the vertebrae under axial compression. The axial strain distributions in the tumour-bearing and matched control vertebrae were investigated by calculating the median, minimum $\left(10^{\text {th }}\right.$ percentile $)$ and maximum $\left(90^{\text {th }}\right.$ percentile $)$ of the strain distribution. Analysis of variance was used to look for statistical differences between mean strain within the tumour bearing vertebrae $\left({\overline{\varepsilon_{z s}}}_{\mathrm{met}}\right)$ and control vertebrae $\left({\overline{\varepsilon_{\mathrm{xg}}}}_{\mathrm{cont}}\right)$, where the vertebra was the experimental unit. (Excel, Microsoft, Redmond WA) The strain fields were also assessed by plotting contours and observing qualitative differences between the metastatic vertebrae and the matched control levels. Post-hoc the relationship between mean bone density and the median strain of the vertebrae was quantified with linear regression.

\section{Results}

\section{Tumour growth}

Metastasis to the vertebral bodies was detectable by bioluminescent imaging at 2-3 weeks post injection. Metastatic tumours detected by dissection were also present in the ovaries, lungs and femurs. The largest spinal tumours were identified in T12, L2 (2), L3, and L4 in the 5 rats. Metastatic involvement was confirmed with $\mu \mathrm{CT}$ imaging. (Figure 2) Higher levels of osteolysis were found to occur near the cranial and caudal ends of the vertebral bodies. The presence of tumour reduced average bone density within the vertebral bodies analyzed by an average of $8 \pm 4 \%$.

\section{Comparison of strain distributions}

Strain distributions were successfully quantified by image correlation in both control and tumour-bearing specimens. (Figure 3) The metastatically involved vertebrae overall experienced an average of 2 times more compressive strain $\left({\overline{\varepsilon_{z s}}}_{m \varepsilon t}=-0.059, \bar{s}_{z=} c_{o n t}=-0.027\right)$ than the control vertebrae $(\mathrm{p}=0.01)$. The mean bone density of the vertebrae was not found to strongly correlate with median strain $\left(\mathrm{R}^{2}=0.3, \mathrm{p}=0.1\right)$.

In all vertebrae the growth plates and adjacent trabecular bone was a site of strain concentration. The growth plate appeared to be nearly completely calcified in all specimens; however a small amount of compression can be seen by inspection of the loaded and unloaded scans. (Figure 4, Figure 5) This is reflected in the increased axial strain measured in this area. The sets of matched lumbar vertebrae all had small regions of tensile strain present $(\sim 0.005$ strain, Figure 3), while the one set of thoracic vertebrae experienced entirely compressive strain.

In four of the five tumour-bearing vertebrae strain concentrations were observed in trabecular regions adjacent to the dorsal wall. (Figure 4) These strain concentrations were not seen in the vertebral level matched controls.

The application of axial compressive loading of all tumour-bearing vertebrae and 4 of the 5 matched controls was consistent, based on the direction and position of the adjacent vertebrae, which applied the load to the vertebra of interest. (Figure 4, Figure 5) One of the control vertebrae (\#4) showed a difference in the alignment of the vertebrae yielding a distinct loading profile with the caudal vertebra positioned more dorsally. Not surprisingly, this vertebra demonstrated a different strain distribution due to the asymmetry in load application relative to 
the dorsal-ventral axis, with greater average strain, particularly along the dorsal wall when compared to the other control vertebrae.

\section{Discussion}

This study demonstrates the successful application of $\mu \mathrm{CT}$-based image registration for the purpose of observing the biomechanical implications of osteolytic metastatic disease within rodent vertebrae through calculation of strain fields. In comparing tumour bearing vertebrae against level matched controls, it is clear that strain differences can be resolved with the developed image registration algorithm. The $\mu \mathrm{CT}$-based image registration method allowed for the assessment of trabecular strain in its native whole bone environment, representing an advance over earlier studies focused on bone cores.

Image registration was able to highlight regions of high strain found in the metastatic vertebrae adjacent to the dorsal wall, a pattern not seen in the matched controls. The hypothesis that strain distributions within this rat model would be consistent with known fracture patterns for osteolytic metastases were confirmed by two observations: 1 . Metastatically involved vertebrae were found to experience greater magnitudes of strain. These higher strains in the metastatically involved vertebrae are in accordance with previous work quantifying the architectural impact and mechanical strength of metastatically compromised vertebrae. ${ }^{6,25-28,38-41,24} 2$. The strain concentrations were found to occur adjacent to the dorsal vertebral body wall, consistent with clinical burst fracture patterns (failure of the posterior vertebral body wall). ${ }^{8,29}$ The strain concentrations on the dorsal wall align with areas of osteolytic destruction. These elevated strain measures are consistent with previous experimental and computational analyses demonstrating that areas with low bone density lead to decreases in structural rigidity and reduced strength, resulting in increased fracture risk in bones with osteolytic skeletal lesions. ${ }^{10,30, \underline{43}}$. Finite element modelling of tumour involved vertebrae has similarly shown mechanical behaviour consistent with increased risk of burst fracture initiation. ${ }^{4,5,7-9,26-28,31}$

Within this study the osteolytic involvement was not strongly correlated with the average strain within the vertebrae. The strain concentrations that were calculated by image registration were not always co-localized with focal defects as visualized on the $\mu \mathrm{CT}$ images. Previous histologic analyses conducted in this rodent model, however, have shown both focal and diffuse metastatic involvement within dorsal regions of the vertebrae. ${ }^{32}$ The presence of diffuse disease was beneficial for the algorithm as it provides more texture information with which to measure strain as compared to distinct focal lesions. The lack of direct correspondence may be attributable to the physiological (and complicated) loading applied through the intervertebral discs and the inability of $\mu \mathrm{CT}$ to identify the presence and impact of diffuse tumour involvement, prior to sufficient bone resorption creating focal defects.

Translation of whole bone biomechanical findings from rodent models to the adult human spine must be approached cautiously due to differences in morphology. Rat vertebrae have distinct geometry in which the vertebral bodies are much longer, with much greater cortical shell thickness relative to the amount of trabecular bone as compared to human vertebrae. In addition, the growth plates are present within rat vertebrae throughout their life cycle. In our work, the growth plates and adjacent trabecular regions were shown to absorb high amounts of strain in the rat vertebral bodies. The growth plates may explain some of the large strains calculated in the vertebrae. This is in agreement with previous findings based on application of this algorithm in 
healthy vertebrae that the presence of the growth plate causes strain concentrations in surrounding trabecular bone. ${ }^{20}$ A study looking at older rats with more highly calcified growth plates $^{33}$, may better elucidate this issue, however this may not be possible concurrently with the study of metastatic disease, as immune-deficient rats develop resistance to metastatic involvement as they age.

The mechanical jig used to apply load to the spinal motion segments was stable, allowing the application of relatively high loads sufficient to yield large, measurable strains within the vertebrae. While the magnitude of these strains, challenges the assumptions of infinitesimal strain theory, the estimated average error from making the approximations of the infinitesimal theory were estimated to be on the order of 0.0075 strain. As well, the magnitude of the measured strains suggests that damage may have occurred within the vertebrae. Structural damage (i.e. breaking trabeculae) could potentially lead to errors in strain measurement, no macroscopic damage was detected after scanning was complete. Microdamage (in the form of linear cracks and diffuse patterns, which consistently occurs within bone) ${ }^{34}$ in metastatically involved bone is the content of ongoing research within the laboratory. The presence of high strains leading to damage may present additional challenges in translating this work to an in vivo model.

The loading was applied to each spinal motion segment, to better reflect physiologic boundary conditions. Utilization of a physiologic boundary condition was thought to be of particular importance in this analysis as strain distributions calculated by digital image correlation have been found to be sensitive to subtle changes in boundary conditions and loading through the intervertebral discs is important clinically in the initiation of burst fractures. ${ }^{35}$ The device produced repeatable axial compressive loading, with the exception of the single control specimen, which exhibited a distinct vertebral malalignment. This malalignment led to loading that was not purely axial compressive, and resulted in a wider distribution of strain, both in tension and compression. The removal of the posterior element/facet joint articulations may have contributed to this alignment shift during axial compressive loading. Modifications to the loading device and the retention of the posterior elements in future testing may allow for more physiologic loads to be realized and interpreted, albeit under a more complex loading scenario. The presence of metastatic involvement of the posterior or transverse elements may also affect the boundary conditions applied in this and future investigations. Strain distribution is critical in understanding bony stability. ${ }^{36}$ Additional work using this algorithm may examine the relationship between the spatial distribution of tumour and the strain distribution to identify more problematic tumour configurations and effective treatments. ${ }^{37}$

Given the uniaxial loading protocol, the largest strain values were expected in the axial direction and as such axial strains could be measured with the least error. This increase in accuracy and the limits in statistical power that would come from multiple comparisons were seen to outweigh any increased insight from the determination of other strains (principal, von Mises, etc.) derived from tensor components.

Strain distributions yield important information which can lend insight into mechanisms of instability and as such the development and targeting of appropriate interventions. ${ }^{38}$ Strain measurement by image registration may be particularly suited to studying interventions that create difficulty for FE analysis due to unknown material properties or boundary conditions. Yerby has used image registration to show the importance of subtle changes in boundary conditions to the strain experienced by trabecular bone cores. ${ }^{35}$ Similarly McKinley used image 
correlation to show the importance of subtle changes in soft tissue boundary conditions on bone strains. ${ }^{39-41}$ Toh, using a $2 \mathrm{D}$ implementation of texture correlation examined a possible mechanism for wedge fracture in osteoporotic thoracic vertebra, showing the strain increased in the anterior of vertebrae during flexion. ${ }^{42}$

Image registration yields a strain field, including magnitude and distribution of strain without assumptions about the magnitude or distribution of applied loading or inherent bone or soft tissue material properties. However, the spatial resolution of strain measurement possible with this image registration technique is limited based on the texture of the material being analyzed. ${ }^{11,19}$ The strain distributions calculated are consequently lower resolution than the images from which they are derived, as the calculation relies on tracking regions of the trabecular structure. A trade-off exists between the size of the regions used and the resolution of the strain field. A larger region results in greater precision but less accuracy, due to smoothing of the strain field calculated when the field varies in space. A consequence of the limited resolution of the strain calculations was the increased strain in the growth plates lead to adjacent bone appearing highly strained ${ }^{43}$ and the precise localization of strain maxima (i.e. adjacent to the dorsal wall) is restricted. The low resolution of the strain calculation means that the underlying strain field was undersampled and hence the exact discretization that was used in the calculation had an effect on the exact position and magnitude of strains calculated. This undersampling may explain some of the discontinuities within the strain field. (Figure 4)

The subset size within this study varied based upon the size of the specimen. Previous investigators have found that the precision of strain calculated by digital volume correlation (a technique similar to the one presented here) was weakly related to changes in the size of the subset. ${ }^{11}$ The standard deviation of the strain calculated was decreased by $35 \%$ when doubling the linear size of the estimating volume. Extrapolating the result from the literature to the variation in subset size present within the investigation presented here implies that the change in linear size of the subsets would result in a difference in precision of roughly $5 \%$.

This study demonstrates the ability of image registration to demonstrate both quantitative and qualitative differences in the biomechanical response of thoracolumbar rat vertebrae due to osteolytic metastatic involvement. Qualitatively, tumour involvement was seen to cause greater strain concentrations near the posterior vertebral body wall, reflective of posterior wall compromise typically associated with osteolytic spinal disease. The success of this application motivates the use of image-based registration to examine the effectiveness of systemic and targeted treatments in reducing strains (and fracture risk) in pathologic bones. Future comparisons of strain distributions under simple and more complex physiologic loading scenarios using this protocol, pre and post treatment, may allow detailed quantified assessments to be performed on the efficacy of interventions directed at spinal metastases.

Acknowledgements: Support for this study was received from the Canadian Institutes for Health Research and the Canadian Breast Cancer Foundation, Ontario Chapter.

\section{References}

1. Weigelt, B, Peterse, JL, van't Veer, LJ. 2005. Breast cancer metastasis: markers and models. Nat Rev Cancer 5: 591-602. 
2. Wong, DA, Fornasier, VL, MacNab, I. 1990. Spinal metastases: the obvious, the occult, and the impostors. Spine 15: 1-4.

3. Ortiz Gomez, JA. 1995. The incidence of vertebral body metastases. International orthopaedics 19: 309-311.

4. Mizrahi, J, Silva, MJ, Hayes, WC. 1992. Finite element stress analysis of simulated metastatic lesions in the lumbar vertebral body. Journal of Biomedical Engineering 14: 467-475.

5. Roth, SE, Mousavi, P, Finkelstein, J, et al. 2004. Metastatic burst fracture risk prediction using biomechanically based equations. Clinical Orthopaedics \& Related Research: 83-90.

6. Ebihara, H, Ito, M, Abumi, K, et al. 2004. A biomechanical analysis of metastatic vertebral collapse of the thoracic spine: a sheep model study. Spine 29: 994-999.

7. Whyne, CM, Hu, SS, Lotz, JC. 2003. Biomechanically derived guideline equations for burst fracture risk prediction in the metastatically involved spine. Journal of Spinal Disorders \& Techniques 16: 180-185.

8. Whyne, CM, Hu, SS, Lotz, JC. 2003. Burst fracture in the metastatically involved spine: development, validation, and parametric analysis of a three-dimensional poroelastic finite-element model. Spine 28: 652-660.

9. Tschirhart, CE, Nagpurkar, A, Whyne, CM. 2004. Effects of tumor location, shape and surface serration on burst fracture risk in the metastatic spine. Journal of Biomechanics 37: 653660.

10. Snyder, BD, Hauser-Kara, DA, Hipp, JA, et al. 2006. Predicting fracture through benign skeletal lesions with quantitative computed tomography. Journal of Bone and Joint Surgery-American Volume 88A: 55-70.

11. Verhulp, E, van Rietbergen, B, Huiskes, R. 2004. A three-dimensional digital image correlation technique for strain measurements in microstructures. Journal of Biomechanics 37: 1313-1320.

12. Smith, TS, Bay, BK, Rashid, MM. 2002. Digital Volume Correlation Including Rotational Degrees of Freedom during Minimization. Experimental Mechanics 42: 272-278.

13. Zauel, R, Yeni, YN, Bay, BK, et al. 2006. Comparison of the linear finite element prediction of deformation and strain of human cancellous bone to $3 \mathrm{D}$ digital volume correlation measurements. Journal of Biomechanical Engineering 128: 1-6.

14. Bay, BK, Smith, TS, Fyhrie, DP, Saad, M. 1999. Digital Volume Correlation: Threedemensional Strain Mapping Using x-ray Tomography. Experimental Mechanics 39: 217-226.

15. Liu, L, Morgan, EF. 2007. Accuracy and precision of digital volume correlation in quantifying displacements and strains in trabecular bone. Journal of Biomechanics 40: 3516-3520.

16. Bevill, G, Eswaran, SK, Gupta, A, et al. 2006. Influence of bone volume fraction and architecture on computed large-deformation failure mechanisms in human trabecular bone. Bone 39: 1218-1225.

17. Eswaran, SK, Bayraktar, HH, Adams, MF, et al. 2007. The micro-mechanics of cortical shell removal in the human vertebral body. Computer Methods in Applied Mechanics and Engineering 196: 3025-3032.

18. Bay, B. 2008. Methods and applications of digital volume correlation. The Journal of Strain Analysis for Engineering Design 43: 745-760.

19. Hardisty, MR, Whyne, CM. 2009. Whole bone strain quantification by image registration: a validation study. J Biomech Eng 131: 064502. 
20. Hardisty, M, Akens, M, Yee, A, Whyne, C. 2010. Image Registration Demonstrates the Growth Plate has a Variable Affect on Vertebral Strain. Annals of Biomedical Engineering 38: 2948-2955.

21. Akens, MK, Hardisty, MR, Wilson, BC, et al. 2009. Defining the therapeutic window of vertebral photodynamic therapy in a murine pre-clinical model of breast cancer metastasis using the photosensitizer BPD-MA (Verteporfin). Breast Cancer Res Treat 119: 325-333.

22. Hardisty, M, Gordon, L, Agarwal, P, et al. 2007. Quantitative characterization of metastatic disease in the spine. Part I. Semiautomated segmentation using atlas-based deformable registration and the level set method. Med Phys 34: 3127-3134.

23. Whyne, C, Hardisty, M, Wu, F, et al. 2007. Quantitative characterization of metastatic disease in the spine. Part II. Histogram-based analyses. Med Phys 34: 3279-3285.

24. Won, E, Akens, MK, Hardisty, MR, et al. 2010. Effects of Photodynamic Therapy on the Structural Integrity of Vertebral Bone. Spine 35: 272-277 210.1097/BRS.1090b1013e3181af8360.

25. Hong, J, Cabe, GD, Tedrow, JR, et al. 2004. Failure of trabecular bone with simulated lytic defects can be predicted non-invasively by structural analysis. Journal of orthopaedic research : official publication of the Orthopaedic Research Society 22: 479-486.

26. Silva, MJ, Hipp, JA, McGowan, DP, et al. 1993. Strength reductions of thoracic vertebrae in the presence of transcortical osseous defects: effects of defect location, pedicle disruption, and defect size. European spine journal : official publication of the European Spine Society, the European Spinal Deformity Society, and the European Section of the Cervical Spine Research Society 2: 118-125.

27. McGowan, DP, Hipp, JA, Takeuchi, T, et al. 1993. Strength reductions from trabecular destruction within thoracic vertebrae. Journal of spinal disorders 6: 130-136.

28. Dimar, JR, 2nd, Voor, MJ, Zhang, YM, Glassman, SD. 1998. A human cadaver model for determination of pathologic fracture threshold resulting from tumorous destruction of the vertebral body. Spine 23: 1209-1214.

29. Denis, F. 1983. The 3 Column Spine and Its Significance in the Classification of Acute Thoracolumbar Spinal-Injuries. Spine 8: 817-831.

30. Snyder, BD, Cordio, MA, Nazarian, A, et al. 2009. Noninvasive prediction of fracture risk in patients with metastatic cancer to the spine. Clinical Cancer Research 15: 7676.

31. Tschirhart, CE. 2005. Biomechanics and Prophylactic Treatment of Spinal Metastases: A Finite Element Based Study. University of Toronto, Toronto.

32. Won, E, Wise-Milestone, L, Akens, M, et al. 2010. Beyond bisphosphonates: photodynamic therapy structurally augments metastatically involved vertebrae and destroys tumor tissue. Breast Cancer Research and Treatment 124: 111-119.

33. Villemure, I, Cloutier, L, Matyas, JR, Duncan, NA. 2007. Non-uniform strain distribution within rat cartilaginous growth plate under uniaxial compression. Journal of Biomechanics 40: 149-156.

34. Martin, R. 2002. Is all cortical bone remodeling initiated by microdamage? Bone 30: 8-13.

35. Yerby, SA, Bay, BK, Toh, E, et al. 1998. The effect of boundary conditions on experimentally measured trabecular strain in the thoracic spine. Journal of Biomechanics 31: 891-897. 
36. Tschirhart, CE, Finkelstein, JA, Whyne, CM. 2006. Metastatic burst fracture risk assessment based on complex loading of the thoracic spine. Annals of Biomedical Engineering 34: 494-505.

37. Tschirhart, CE, Finkelstein, JA, Whyne, CM. 2006. Biomechanics of vertebral level, geometry, and transcortical tumors in the metastatic spine. Journal of Biomechanics.

38. Babb, A, Carlson, WO. 2006. Vertebral compression fractures: treatment and evaluation. S.D.Med. 59: 343-345, 347.

39. Brown, AN, McKinley, TO, Bay, BK. 2002. Trabecular Bone Strain Changes Associated With Subchondral Bone Defects of the Tibial Plateau. Journal of Orthopaedic Trauma 16: 638-643.

40. McKinley, TO, Bay, BK. 2001. Trabecular bone strain changes associated with cartilage defects in the proximal and distal tibia. Journal of orthopaedic research : official publication of the Orthopaedic Research Society 19: 906-913.

41. McKinley, TO, English, DK, Bay, BK. 2003. Trabecular bone strain changes resulting from partial and complete meniscectomy. Clin Orthop Relat Res: 259-267.

42. Toh, E, Yerby, SA, Bay, BK, et al. 2005. The behavior of thoracic trabecular bone during flexion. The Tokai journal of experimental and clinical medicine 30: 163-170.

43. Shi, X, Wang, X, Niebur, G. 2009. Effects of Loading Orientation on the Morphology of the Predicted Yielded Regions in Trabecular Bone. Annals of Biomedical Engineering 37: 354-362. 
1

2

3

4

5

6

7

8

9

10

11

12

13

14

15

16

17

18

19

20

21

22

23

24

25

26

27

28

29

30

31

32

33

34

35

36

37

38

39

40

41

42

43

44

45

46

47

48

49

50

51

52

53

54

55

56

57

58

59

60

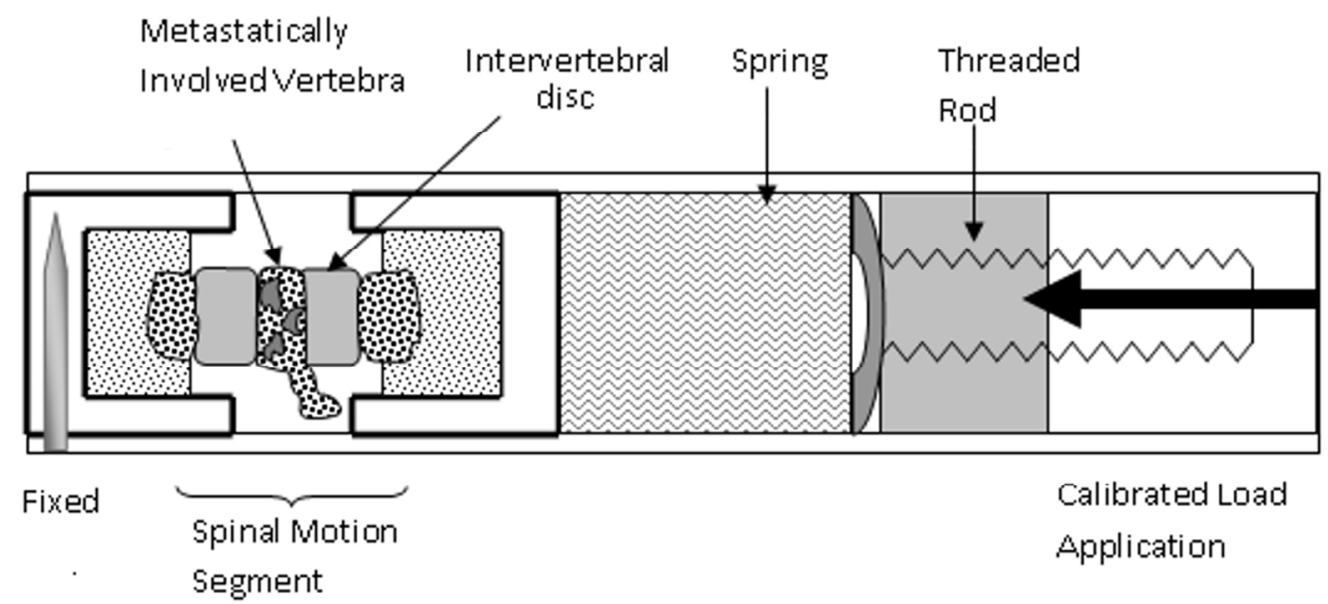

Figure 1: Spring-loaded tubular axial compressive loading device. Axial load is ensured by the tubular design in which specimens are fixed to acetal rings that slide within an outer polycarbonate tube. Load is applied by shortening the spring within the device. The vertebrae adjacent to the level of interest were fixed to the loading device with bone cement (Simplex, Stryker, Kalamazoo, USA). Force was applied to the

vertebra of interest via the adjacent vertebral bodies and intervertebral discs. The distal parts of the posterior elements of the adjacent vertebrae were trimmed prior to potting in bone cement to remove facet joint loading.

$97 \times 44 \mathrm{~mm}(300 \times 300$ DPI $)$

John Wiley \& Sons, Inc. 

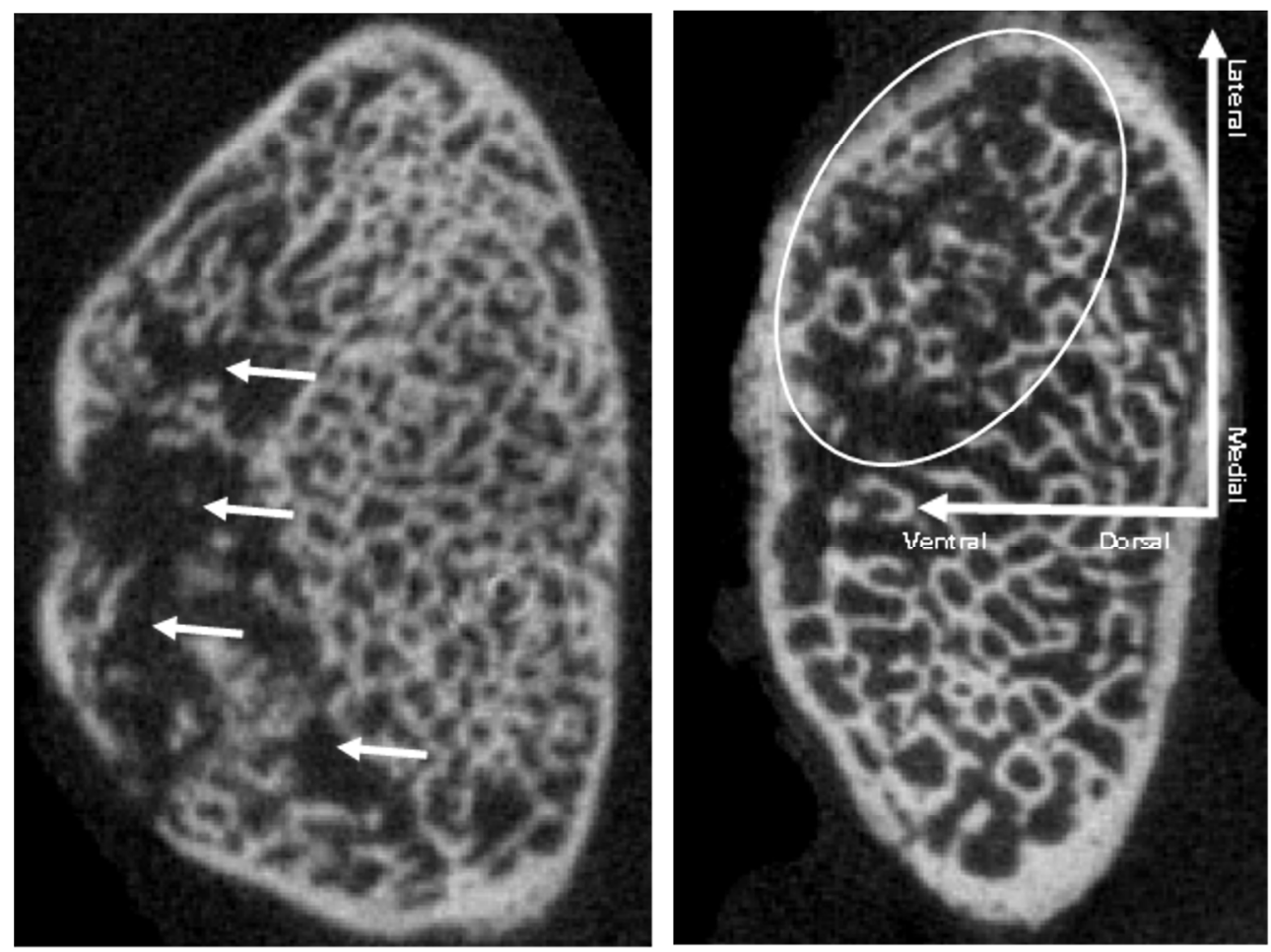

Figure 2: Examples of metastatic tumours in rat vertebral bodies. The tumours were created by the intercardiac injection of MT-1 human breast cancer cells in rnu/rnu rats. The left image depicts a vertebral body with distinct focal lesions near the ventral wall of the vertebral body (identified by white arrows). The right image depicts a vertebral body with more diffuse metastatic involvement (region identified by the white ellipse).

$203 \times 148 \mathrm{~mm}(300 \times 300 \mathrm{DPI})$ 
Figure 3: Box Plot of of axial strain distributions for tumour-bearing vertebrae and vertebral level matched controls, calculated by image registration. Standard box plot parameters are depicted, whiskers are the 10th and 90th percentiles, the box size is the 25th and 75th percentiles and the heavy dark band is the median of the distribution. The graph demonstrates that both groups of specimens contain large nonnormal distributions of strain. The tumour bearing vertebrae have a greater percentage of highly strained bone which is consistent with statistically significant difference found between the tumour and control vertebral means. All of the tumour bearing vertebrae have greater ranges of strain (25-75\%) than their level matched controls. $101 \times 112 \mathrm{~mm}(300 \times 300$ DPI $)$ 

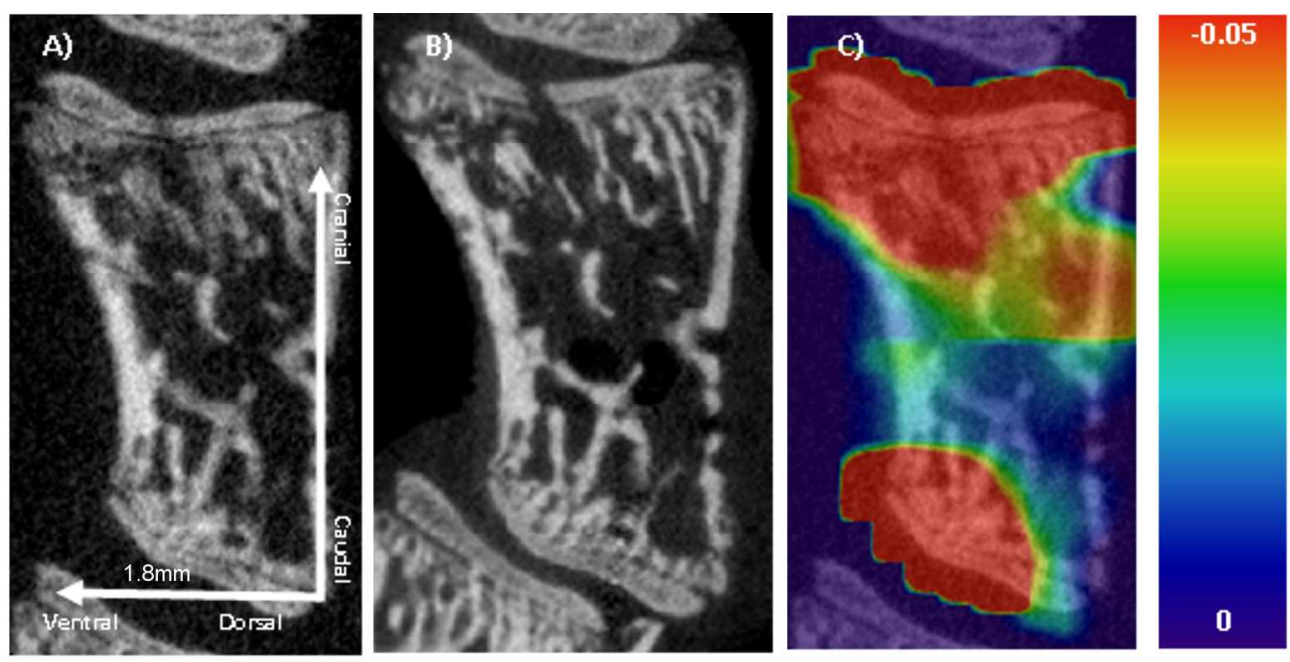

$164 \times 82 \mathrm{~mm}(300 \times 300 \mathrm{DPI})$ 

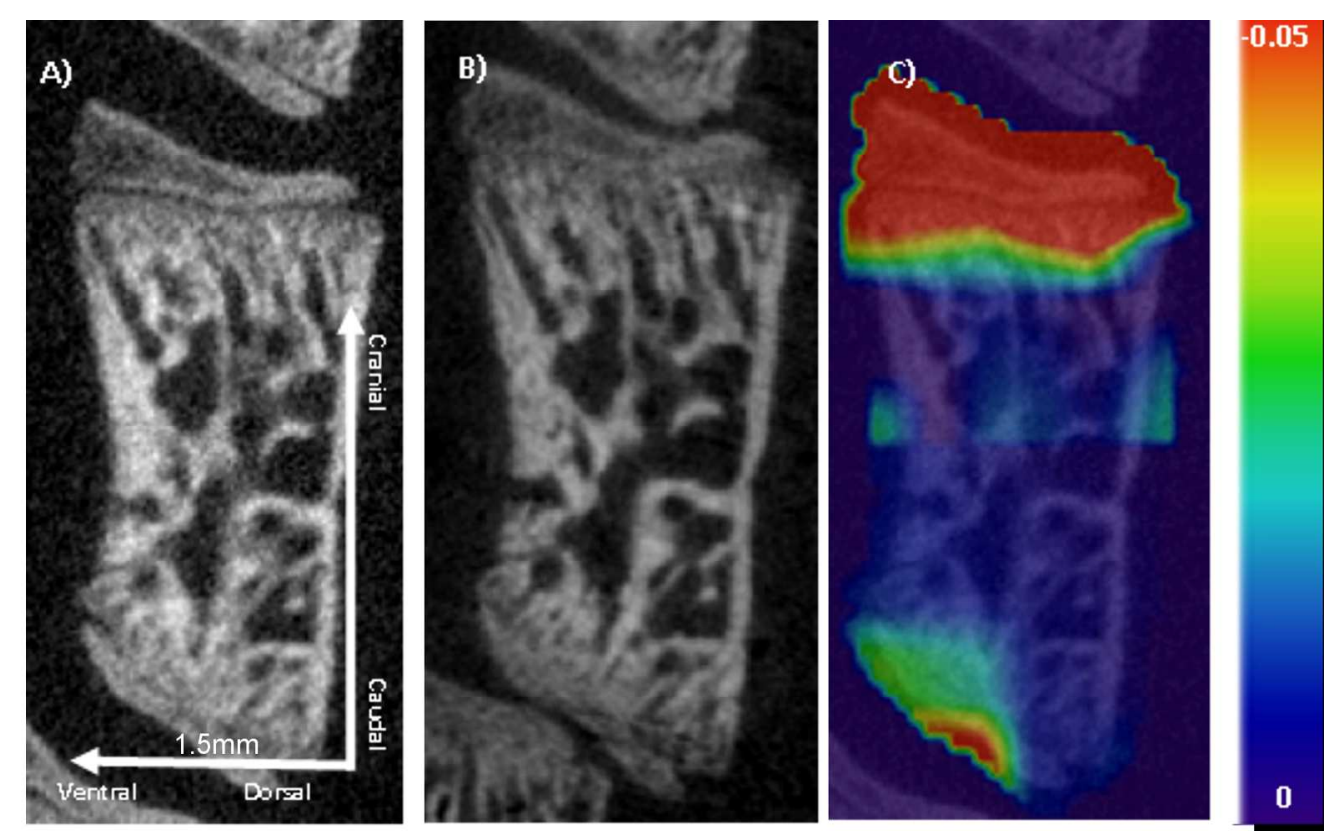

$173 \times 108 \mathrm{~mm}(300 \times 300$ DPI)

John Wiley \& Sons, Inc. 